\title{
An Improved Computational Model for Adaptive Communication Channel Estimation
}

\author{
S.A. Akinboro, G.A. Aderounmu, E.A. Olajubu \\ A.O. Ajayi, I.K. Ogundoyin, O.M Olaniyan
}

\author{
S.A. Akinboro, O.M Olaniyan \\ Computer Science and Technology \\ Bells University of Technology, Ota, Nigeria \\ E-mail: akinboro2002@yahoo.com
}

\author{
G.A. Aderounmu, E.A. Olajubu, A.O. Ajayi, I.K. Ogundoyin \\ Computer Science and Engineering \\ Obafemi Awolowo University, Ile-Ife, Nigeria
}

\begin{abstract}
Channel estimation is an important and necessary function performed by modern wireless receivers. The goal of channel estimation is to measure the effects of the channel on known or partially known transmission. The usual practice in acquiring knowledge about a channel is to model the channel and then acquire the parameters involved in the model. This paper proposes a variable partial update model for adaptive communication channel estimation with a view to improving signal error at the receiver station. The proposed model is composed of finite impulse response transversal adaptive filter and least mean square adaptation algorithm. The performance of the proposed model was compared with the full update model. The evaluation results indicated that the proposed model performed better than the full update model in terms of computational complexity, memory load, and convergence rate.
\end{abstract}

Keywords: Adaptation algorithm, Computational complexity, Memory load, convergence rate, Partial update

\section{Introduction}

Channel refers to one way telecommunication link or transmission medium through which information or signal is transmitted from a transmitter to a receiver. It may either be physical or logical depending on the application, for example cables, and radio frequency are physical channels while control and traffic channels within the ratio frequency channel are logical channels. In estimation theory, it is assumed that the desired information is embedded in a noisy signal. Noise adds uncertainty and if there is no uncertainty then there would be no need for estimation. The channel deforms the transmitted signals often in unpredictable ways. To retrieve the information that is transmitted, the received signal has to be processed. The retrieval of information about the channel either from the received signal or from the signal sent is known as channel estimation. The major sources of impairment in wireless channels include channel time-variation, Inter Symbol Interference, and Co Channel Interference [6]. In order to deal with these problems, the transmitted signal needs to be processed at the receiver station. A core feature of many modern communication systems is their ability to adapt to the working environment. The technology at the heart of these flexible systems is an adaptive digital filter whose coefficients change in response to external conditions [3]. An adaptive filter has coefficients that are updated by some types of adaptive algorithms to improve or optimize its response to a desired performance. In general, adaptive filters consist of two basic parts: the filter which applies the required processing 
on the incoming signal to be filtered and an adaptive algorithm which adjusts the coefficient of the filter to improve its performance [1]. Many computationally efficient algorithms have been developed for adaptive filtering [2]. They are based on either a statistical approach such as least-mean square (LMS) algorithm or a deterministic approach such as recursive least-square (RLS) algorithm. The major advantage of the LMS algorithm is its computational simplicity. The RLS algorithm conversely, offers faster convergence but with a higher degree of computational complexity. Increased popularity of mobile phones and other wireless communication products provide the demand for developing appropriate techniques to improve the performance of existing system for reliable transmission of data over wireless communicational system. Many applications in wireless communication like channel estimation and echo cancellation require the adaptive filter to have a very large number of co-efficient, and updating the entire coefficient is costly in terms of power, memory and computation, and sometimes impractical for mobile units [2]. In this paper, an improved computational model for adaptive channel estimation is proposed. The adaptive channel estimator is modelled using finite impulse response traversal adaptive filter. The adaptation process of the filtering is performed using Variable Partial Update LMS algorithm. The coefficient of the adaptive filter is partially updated to reduce computational cost and memory load, while at the same time updating the step size parameter to enhance the speed and the accuracy of convergence. The outline of this paper is as follows. In section 2 , the review of related works is carried out, and in section 3, the proposed model is described together with the adaptation algorithm used. Simulation and results are presented in section 4 , and section 5 concludes the study.

\section{Related work}

Several research works have been done in developing computational models and adaptation algorithms for adaptive communication channel estimation but all the models failed to address the issues of convergence rate, memory load and computational complexity efficiently. In particular, no research work has considered the comparison of the full update and the partial update of the adaptive digital filter coefficients using the parameters memory load, convergence rate and computational complexity. In an attempt to reduce computational complexity and improve asymptotic performance, [4] Proposed active tap detection LMS algorithm. Though the research work reduces computational burdens as well as unsatisfactory poor convergence rate asymptotic performance of the adaptive tap in a long channel but storage location is provided for the entire adaptive tap which is quite expensive. Also, [2] proposed partial updating of the LMS adaptive filter to reduce the cost of power, memory load and computation. Sequential partial update LMS is employed in their work. They analyzed the alternating odd/even partial update LMS algorithm and derived stability bound on step size parameter for wide sense stationary and cyclo-stationary signals based on external properties of the matrix 2-norm but comparing with the proposed model, the memory load and computational complexity is still large. The behavior of three variants of variable step size LMS algorithm for training based multi-user detection in a CDMA system was studied by [10]. Two of the algorithm have smaller computational complexity and memory load but still suffers from the fact that their steady state error and speed of convergence depend on the same parameters (the step size), therefore complementary pair variable step size LMS was introduced. Although the proposed algorithm has an increased computational complexity and memory load, but it has better speed performance and more simple parameters setup which are very important in practical applications. A number of previous LMS algorithms were analyzed by [5]. They pointed out their weaknesses and proposed a variant of modified variable step size LMS algorithm which was tested and can ensure convergence in any cases and can provide a higher speed of convergence and a better level of tracking ability. 
The normalized LMS algorithm is adapted to get higher speed of convergence by adjusting the step size through the power of input signals. However, we can hardly make an accurate estimate of the auto correlation matrix and mean square error practically. They also said that variants of variable step size LMS algorithms have been proposed, but in all of them, the step size equation can be written as $\mu(n+1)=\alpha \mu(n)+\gamma p$ where $p$ is a function and depends on the different VSS-LMS algorithm, $\alpha$ and $\gamma$ are constant, $\mu$ is the step size and $\mathrm{n}$ is the time index. Previous research works focused on improving the effect of channel on transmitted signal through proposition of variants of adaptation algorithm and design of improved adaptive digital filters, this work however, compare the performance of full-update and variable partial update variants of the adaptation algorithm with design of adaptive digital filter using index factors. The three performance metrics considered are convergence rate, memory load and computational complexity.

\section{Architecture of the proposed model}

The block diagram for Variable Partial Update LMS Model is applied in this work, Figure 1 depict the model, while the adaptation module of the proposed model is an improvement on [2] which is depicted in Figure 2. The difference between the model developed in [2] and this work is the updates of the coefficients. This model uses index factors of three and five for updates while [2] uses alternate even and odd updates of the coefficients The model consists of four major modules vis-ŕ-vis; unknown channel, FIR filter, summer $\left(\sum\right)$, and adaptation algorithm. The unknown channel is positioned parallel to the FIR filter so that the same input signal can be transmitted simultaneously. The estimation is adaptive and parallel, this is because in wireless situation the paths that a signal takes between the transmitter and the receiver may keep changing. The signal transmitted through the unknown channel is the desired training signal $d(k)$, while the signal transmitted through the FIR filter output is the estimated training signal $y(k)$. The two signals are transmitted through the summer to give the estimated error signal $e(k)$. The estimated error signal is then used to update the coefficient of the FIR filter using the adaptation algorithm called Variable step size Partial Update Least Mean Square algorithm (VPU-LMS) [6].

It is assumed that the variable partial update LMS filter in fig 2 is a standard transversal FIR filter of length $L \geq 5$. Let $\left(x_{i, n}\right)$ be the input sequence and let $\left(w_{i, n}\right)$ denote the coefficients of the adaptive filter.

$W n=[w 1, n, w 2, n w 3, n \ldots w L, n]^{T}$

$X n=[x 1, n, x 2, n x 3, n \ldots x L, n]$

Where the terms define above are for the instant $\mathrm{n}$ and $\mathrm{T}$ denotes the transpose operator. Also, let $d(n)$ denotes the desired response. It represents a known training signal which is transmitted over a noisy channel with unknown FIR transfer function. It was assumed that $d(n)$ obeys a FIR model given by

$$
d(n)=x_{n}+k(n)
$$

Where $k(n)$ is the autoregressive noise signal that is independent of the input sequence $X_{n}$. We also assumed that the filter coefficient is a mutually exclusive set. The elements of the set are coefficients with index factors of three and five i.e. filter length that is divisible by three and five. Therefore the set $\mathrm{S}$ is defined as $S=\left\{w_{3}, w_{5}, w_{6}, w_{9}, w_{10} \ldots.\right\}$

The proposed algorithm called variable partial update LMS algorithm. The algorithm updates only the coefficient of the adaptive filter with index factors of three and five. It also makes sure that only the active coefficients (i.e. value not equal to zero) are used for the update process. The step size parameter is updated to ensure convergence of the algorithm. The algorithm is described in following steps: 


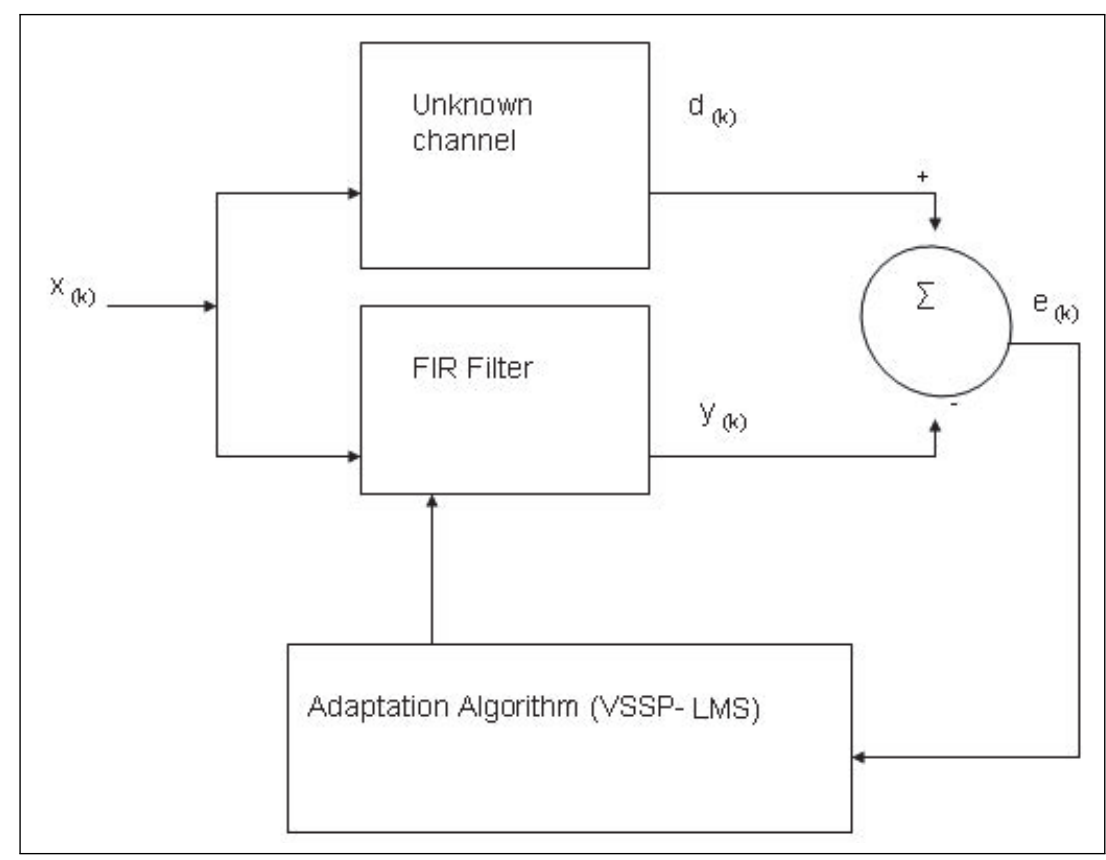

Figure 1: The block diagram for Variable Partial Update LMS Model

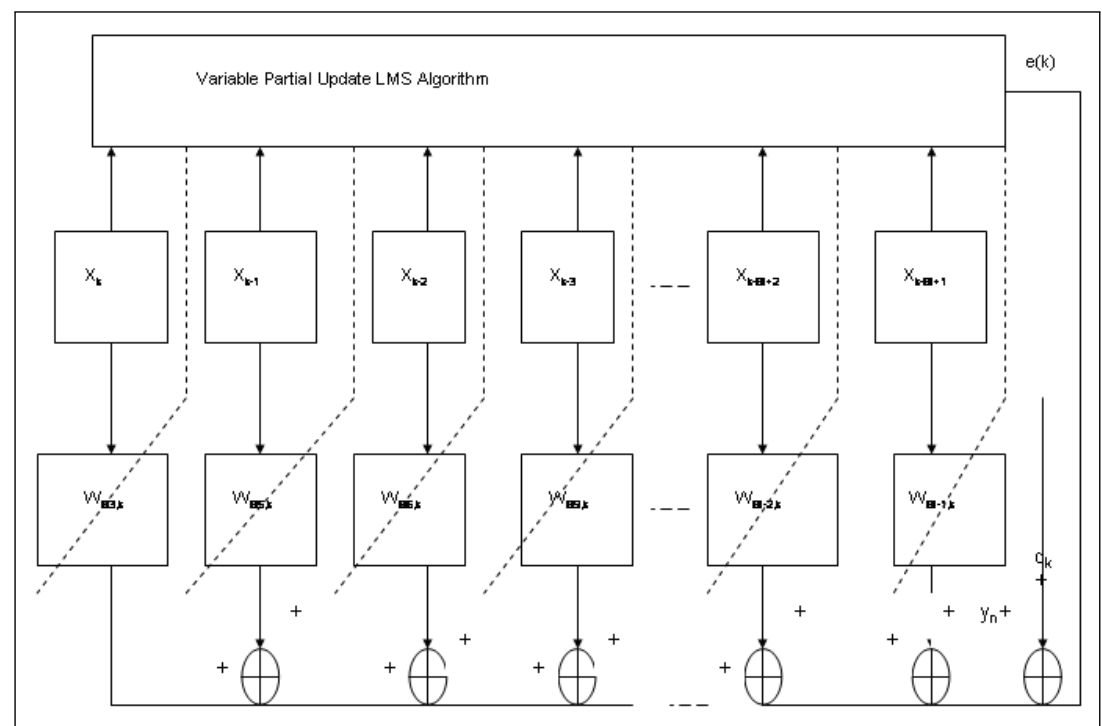

Figure 2: Transversal FIR Filter Structure for the Variable Partial Update-LMS Model 
- Compute the output of the adaptive filter

$$
y(n)=w_{n j}^{T} x_{n j}
$$

where $j \in S$ and $\mathrm{T}$ is the Transpose operator.

- Compute the output error

$$
e(n)=d(n)-y(n)
$$

where $d(n)=x_{n}+K(n)$ is the desired output of the transfer filter

- Update the coefficient of the adaptive filter using Equation(1).

$$
W_{n+1}=\left\{\begin{array}{cl}
W(n-1), j+\mu e(n) * X(n), j & \text { if } j \in S_{i} \text { and } W \neg 0 \\
W_{(n-1), j} & \text { otherwise. }
\end{array}\right.
$$

- Update the step size of the adaptive filter

$$
\begin{gathered}
\mu(n+1)=\alpha \mu(n-1)+\mu(n) p(n) \\
\mu(n+1)=\left\{\begin{array}{cc}
\mu_{\max } & \text { if } j \mu(n+1)>\mu_{\max }, \\
\mu_{\min } & \text { if } j \mu(n+1)<\mu_{\min }, \\
\mu(n+1) & \text { otherwise. }
\end{array}\right. \\
\gamma(n)=\left\{\begin{array}{cl}
\beta \gamma(n-1) & \text { if } j \mu(n+1)>\mu_{\max }, \\
\gamma(n-1) & \text { otherwise }
\end{array}\right.
\end{gathered}
$$

where $\gamma$ and $\beta$ are constant values, $0<\gamma, \beta>1$. The algorithm will adjust the parameter $\gamma$ with the constant $\beta$. To ensure convergence the parameter $\beta$ must satisfy that $0<\beta<1$

\section{Simulation and Results}

To test the performance of the proposed variable partial update LMS algorithm, we simulated the discrete signal sequence generated in Mat lab environment using pseudo random number generator with zero mean and variance of one, and a noise signal sequence which is obtained by introducing 0.8 noise level to the discrete signal. These signals form the desired signals that were input to the finite impulse response filter. The outputs from the filter form the actual signal which was subtracted from the desired signal to obtain the mean square error. To establish the superiority of the proposed partial update model over the full update model, training was performed using fifty different sets of input data with different value of the step size to obtain the average result of the mean square error (MSE) and the efficiency of the two models.

Table 1 shows the values of the step size and other simulation parameters. The full update LMS algorithm and our partial update LMS algorithm were simulated using various values of step size. Figure 3 shows the comparison of the full update and the partial update model

The proposed algorithm exhibit variable update of the step size. In order to test this algorithm for speed and accuracy of convergence, we used the parameters specified in Table 2 for the case when step size is less than the minimum step size (0.0036). Figure 4 shows a specimen comparison of the proposed partial update model with the equivalent full update model.

We illustrate the case when the step size is set to a large value or possibly larger than the maximum step size. Table 3 shows the parameters used for the simulation. In a normal situation, when the mean square error is magnified, stability of the filter is affected. This is 


\begin{tabular}{ccccccc}
\hline $\begin{array}{c}\text { Step } \\
\text { Size Value }\end{array}$ & $\mu_{\max }$ & $\mu_{\min }$ & $\gamma$ & $\alpha$ & $\beta$ & Length \\
\hline 0.001 & 1.9 & 0.001 & 0.0007 & 0.2 & 0.01 & 50 \\
0.0011 & & & & & & \\
0.0013 & & & & & & \\
0.0017 & & & & & & \\
0.0020 & & & & & & \\
0.0030 & & & & & & \\
\hline
\end{tabular}

Table 1: Simulation Parameters for Fixed Step Size $(\mu)$

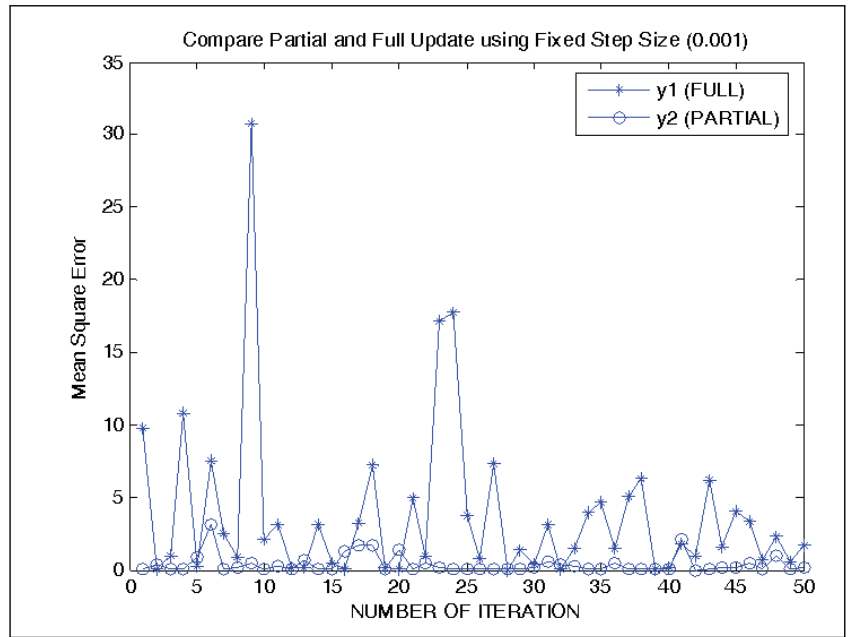

Figure 3: Mean Square Error versus Number of Iteration for Step Size of 0.001

\begin{tabular}{ccccccc}
\hline $\begin{array}{c}\text { Step } \\
\text { Size Value }\end{array}$ & $\mu_{\max }$ & $\mu_{\min }$ & $\gamma$ & $\alpha$ & $\beta$ & Length \\
\hline 0.0036 & 0.09 & 0.01 & 0.0007 & 0.2 & 0.01 & 50 \\
0.0049 & & & & & & \\
0.0052 & & & & & & \\
\hline
\end{tabular}

Table 2: Simulation Parameter for Variable Step Size, for $\left(\mu<\mu_{\min }\right)$

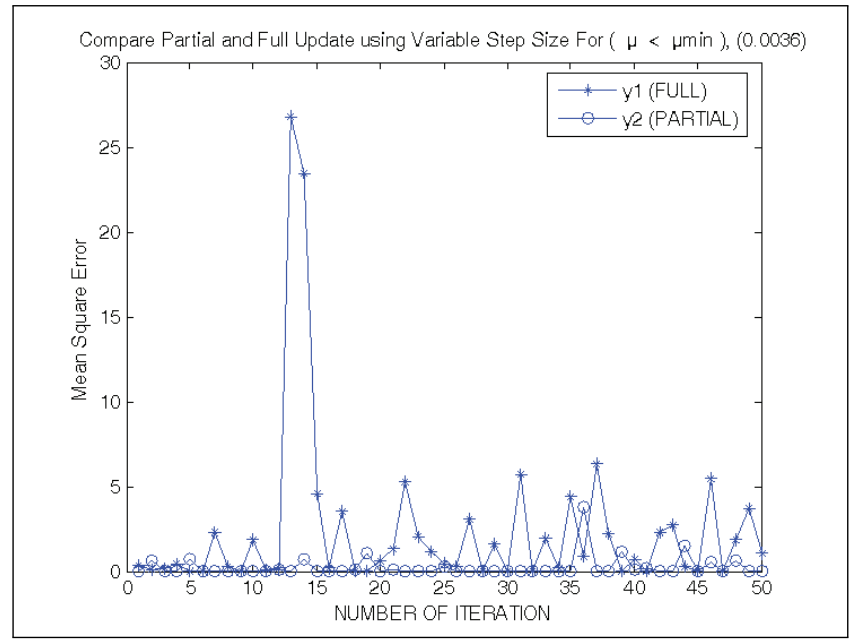

Figure 4: Mean Square Error versus Number of Iteration for $\left(\mu<\mu_{\min }\right)$ 
shown in Figure 5 where the mean square error was magnified and the accuracy of convergence was adversely affected for the full update model. However, the reverse is the case for the partial update model, with the speed and accuracy of convergence still enhanced.

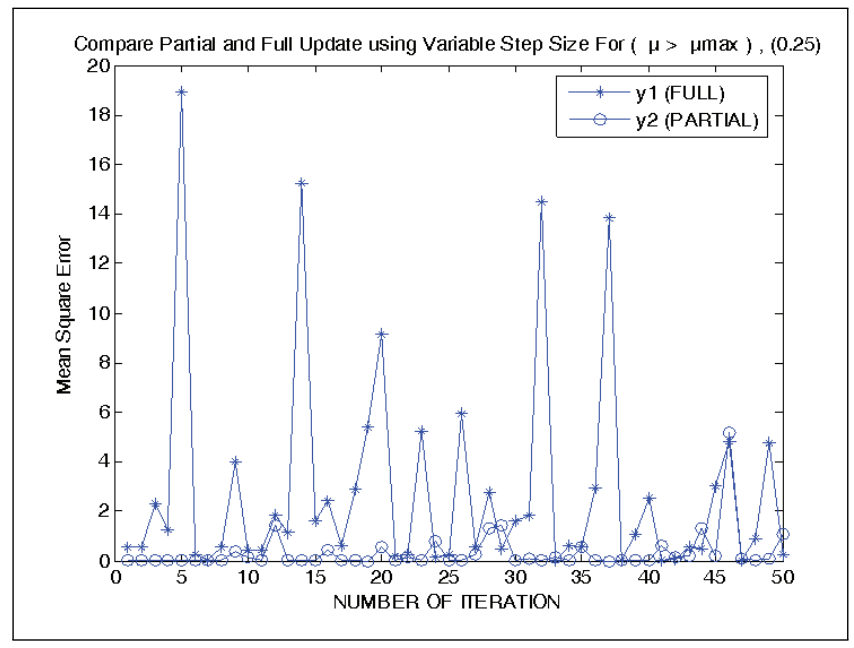

Figure 5: Mean Square Error versus Number of Iteration for $\left(\mu>\mu_{\max }\right)$

\begin{tabular}{ccccccc}
\hline $\begin{array}{c}\text { Step } \\
\text { Size Value }\end{array}$ & $\mu_{\max }$ & $\mu_{\min }$ & $\gamma$ & $\alpha$ & $\beta$ & Length \\
\hline 0.25 & 1.9 & 0.001 & 0.0007 & 0.2 & 0.01 & 50 \\
0.30 & & & & & & \\
0.40 & & & & & & \\
\hline
\end{tabular}

Table 3: Simulation Parameter for Variable Step Size for $\left(\mu>\mu_{\max }\right)$

The filter length was varied with a constant step size to evaluate the system performance. The filter length is equivalent to the number of coefficients required for the update of the filter. The parameter considered here is the memory load. Each of the coefficient requires a unit storage, therefore the lesser the number of coefficient the lesser the memory requirement. For the full update model, all the coefficients are used for the update process therefore the memory load $(M)$ is equivalent to the filter length $(L)$. In the case of partial update model, only the coefficients with index factors of three and five are used for the update. Therefore memory load $M=\frac{L}{3}+\frac{L}{5}$. Where $\frac{L}{3}$ and $\frac{L}{5}$ are the sets of filter length divisible by factors of three and five respectively. Simulation was carried out for fifty iterations using the parameters in Table 4 . The result shown in Figure 6 revealed that the performance of the system is below $50 \%$ for full update model and up to $86 \%$ for the partial update model.

\subsection{Computational Complexity}

Computational complexity refers to the number of hardware resources required to implement the system. The complexity of an algorithm determines the hardware requirement and computational cost. The hardware required to implement the full update and the partial update finite impulse response transversal adaptive filter are the multiplier, summer and the memory. The multiplier is use to multiply the input with the corresponding weight, memory to store the weights, and summer to perform the addition. The computational complexity of our model was estimated by counting the number of hardware resources as described in [12] such as multipliers, 


\begin{tabular}{ccccccccc}
\hline $\begin{array}{c}\text { Filter } \\
\text { Length }\end{array}$ & $\mu_{\max }$ & $\mu_{\min }$ & $\gamma$ & $\alpha$ & $\beta$ & Step size & $\begin{array}{c}\text { Memory Load } \\
\text { Full Update }\end{array}$ & Partial Update \\
\hline 20 & 0.02 & 0.01 & 0.0007 & 0.2 & 0.01 & 0.003 & 20 & 9 \\
50 & & & & & & & 50 & 23 \\
70 & & & & & & & 70 & 33 \\
90 & & & & & & & 90 & 42 \\
100 & & & & & & & 100 & 47 \\
130 & & & & & & & 130 & 61 \\
150 & & & & & & & 150 & 70 \\
170 & & & & & & & 170 & 79 \\
200 & & & & & & & 200 & 93 \\
250 & & & & & & & 250 & 126 \\
\hline
\end{tabular}

Table 4: Simulation Parameter for System Performance Evaluation

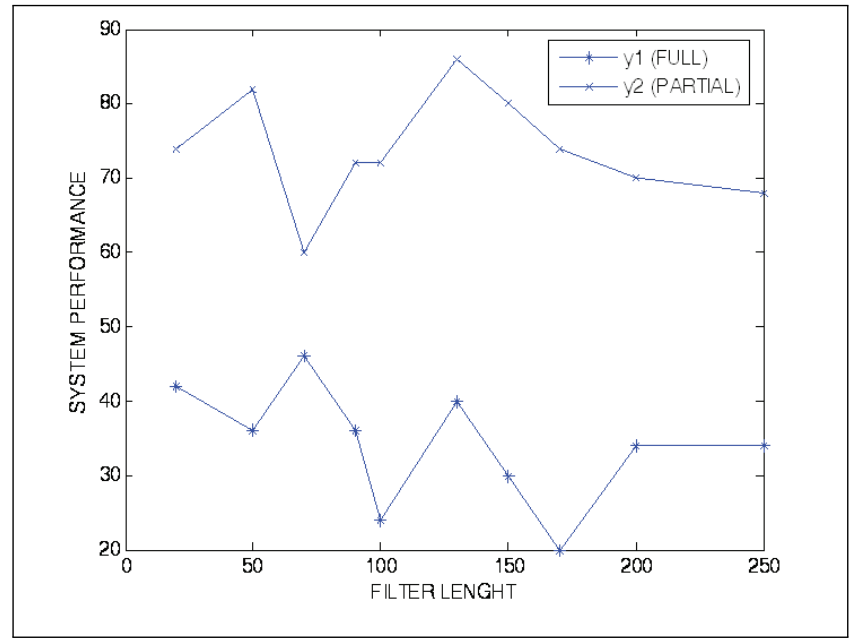

Figure 6: System Performance versus Filter Length 
summers and memories required for a single iteration for each model as shown in Table 5. The evaluation result shown in Figure 7 revealed that the computational complexity of the proposed partial update model is considerably lower when compared with the full update model.

\begin{tabular}{ccccccc}
\hline $\begin{array}{c}\text { Filter } \\
\text { length }\end{array}$ & $\begin{array}{c}\text { Memories } \\
(\mathrm{FU})\end{array}$ & $\begin{array}{c}\text { Summers } \\
(\mathrm{FU})\end{array}$ & $\begin{array}{c}\text { Multipliers } \\
(\mathrm{FU})\end{array}$ & $\begin{array}{c}\text { Memories } \\
(\mathrm{PU})\end{array}$ & $\begin{array}{c}\text { Summers } \\
(\mathrm{PU})\end{array}$ & $\begin{array}{c}\text { Multipliers } \\
(\mathrm{PU})\end{array}$ \\
\hline 20 & 20 & 20 & 20 & 9 & 9 & 9 \\
50 & 50 & 50 & 50 & 23 & 23 & 23 \\
70 & 70 & 70 & 70 & 33 & 33 & 33 \\
90 & 90 & 90 & 90 & 42 & 42 & 42 \\
100 & 100 & 100 & 100 & 47 & 47 & 47 \\
130 & 130 & 130 & 130 & 61 & 61 & 61 \\
150 & 150 & 150 & 150 & 70 & 70 & 70 \\
170 & 170 & 170 & 170 & 79 & 79 & 79 \\
200 & 200 & 200 & 200 & 93 & 93 & 93 \\
250 & 250 & 250 & 250 & 126 & 126 & 126 \\
\hline
\end{tabular}

Table 5: Evaluation of Computational Complexity

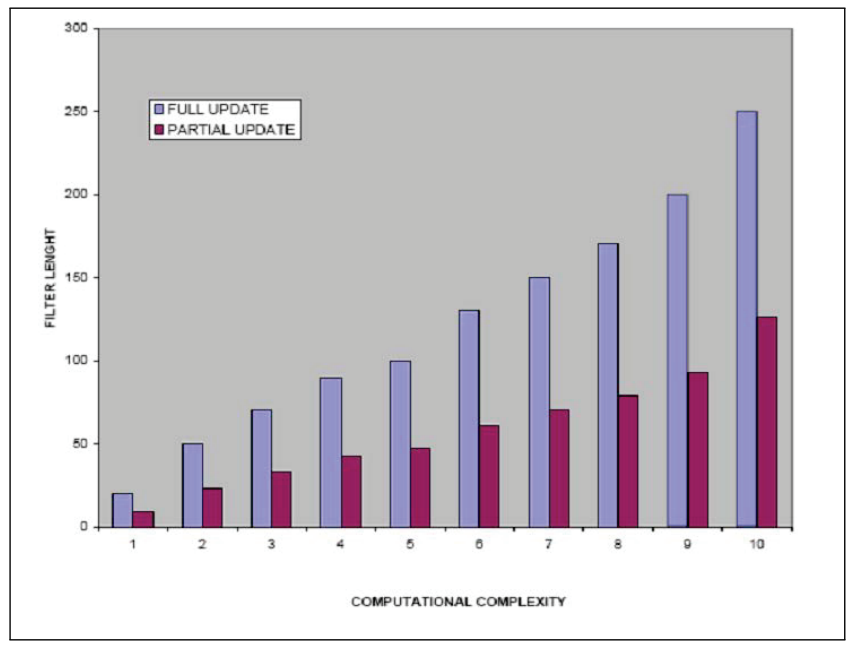

Figure 7: Filter Lengths versus Computational Complexity

\section{Conclusions}

To achieve the continuous update of the coefficient using adaptive algorithm, an improved computational model was proposed in this research, the novelty of which is the adoption of finite impulse response transversal adaptive filter to filter the noise signal from the transmitted signal. The adaptation process employed the concept of variable partial update least mean square algorithm. In the update process, only the coefficients with the factors of three and five are used. The performance of the proposed model was compared with the full update model using the following parameters convergence rate, memory load, and computational complexity in Mat lab environment. The simulation results revealed a better performance of the proposed model over the full update model. The proposed framework will particularly be suitable for wireless communication environment where the characteristics of the channel changes with time. The 
results obtained in the study will go a long way to reducing the effect of channel time variation, inter-symbol interference and co channel interference on the transmitted signal.

\section{Bibliography}

[1] C. S. Douglas, and W. Pan, Exact Expectation Analysis of the LMS Adaptive Filter, IEEE Transaction on Signal Processing, 43(12),2863-2871, 1995.

[2] M. Godavarti and A. O. Hero, Analysis of the Sequential Partial Update LMS Algorithm, Proceedings of IEEE International Conference on Acoustics, Speech, and Signal Processing,pp. 3857-3860, 2001.

[3] S. Mullins and C. Heneghan, Alternative Least Mean Square Adaptive Filter Architectures for Implementation on Field Programmable Gate Arrays, Department of Electronic and Electrical Engineering, University College Dublin available at http://195.134.67.70/eurasip/proceedings/eusipco/2002/articles/paper389.pdf, 2002.

[4] S. Singh, R.K. Bansal, and S. Bansal, Improved Channel Estimation with Auto-Regressive Prewhitening Techniques for Color Inputs, International Conference on Next generation Communication System: ICONGENCOM-06, pp. 9-14, 2006.

[5] Y. Li and W. Xinan, A Modified VS LMS Algorithm, IEEE Transaction on Signal Processing, Vol. 2, No. 5, 615-618, 2005.

[6] S. Diggavi, B. Chong, and A. Paulraj, An Interference Suppression Scheme with Joint Channel Data Estimation, IEEE Journal on Communication, Vol. 17, No.11,465-469, 1998.

[7] J. Sanubari, Fast Convergence LMS Adaptive Filters Employing FuzzyPartial Updates, IEEE Transaction on Signal Processing, Vol. 4, pp. 1334-1337, 2003.

[8] S. Jo, J. Choi and Y. Lee, Modified Leaky LMS Algorithm for Channel Estimation in DSCDMA System, IEEE Communications Letters, Vol. 6, No 5, 202-204 2002.

[9] M. Gadhiok, Channel Estimation for Fast Fading Environment, IEEE Transaction on Signal Processing, Vol. 19, pp. 14-22, 2004.

[10] K. Egiazarian, P. Kuosmanen, and R. C. Bilcu, Variable Step Size LMS Adaptive Filters for CDMA Multiuser Detection, Vol.17 pp. 259-264, 2004.

[11] R. Bilcu, P. Kuosmanen and C. Rusu, A Novel Complementary Variable Step LMS Algorithm, IEEE Transaction on Signal Processing, Vol. 23 pp. 13-19, 2000.

[12] V. Debrunner and D. Zhou, Hybrid Filtered Error LMS Algorithm: Another Alternative to Filtered-x LMS, IEEE Transactions on Circuit and System, Vol. 53, No. 3,pp.653-661, 2006. 